\title{
De vuelta a la clínica. Métodos I. Diseños de investigación. Mayor calidad de información, mayor certeza a la respuesta
}

\author{
Juan O. Talavera, ${ }^{1 *}$ Ivonne Roy-García, ${ }^{2}$ Lino Palacios-Cruz, ${ }^{3}$ Rodolfo Rivas-Ruiz, ${ }^{2}$ Irma Hoyo y \\ Marcela Pérez-Rodríguez ${ }^{2}$ \\ ${ }^{1}$ Dirección de Enseñanza e Investigación, Centro Médico ABC; ${ }^{2}$ Instituto Mexicano del Seguro Social, Coordinación de Investigación en Salud, \\ Centro Médico Nacional Siglo XXI, Centro de Adiestramiento en Investigación Clínica; 'Instituto Nacional de Psiquiatría "Dr. Ramón de la Fuente", \\ Subdirección de Investigaciones Clínicas, Departamento Epidemiología Clínica; ${ }^{4}$ Servicio de Medicina Interna, Centro Médico ABC. Ciudad de \\ México
}

\section{Resumen}

Los diseños de investigación se refieren a la forma como se obtiene la información y están limitados por viabilidad ética, económica y temporal. Son estrategias estandarizadas para disminuir los sesgos que en el modelo arquitectónico de la investigación se identifican en el estado basal, maniobra y desenlace; de ahí que no hay diseños específicos para cada pregunta. El diseño con menor probabilidad de sesgos es el ensayo clínico, seguido de la cohorte, el estudio de casos y controles y, finalmente, la encuesta transversal. Entre las principales características que dan mérito a los diseños están las siguientes: la pesquisa de la población, que se refiere a la ubicación de la población en relación con el curso clínico o historia natural de la enfermedad; la maniobra, o acción que se espera modifique la condición basal, que puede ser observacional o experimental; el seguimiento, o monitoreo documentado que se le da a cada sujeto, que puede ser longitudinal o transversal; y la direccionalidad, prolectiva o retrolectiva, que alude al tiempo de recopilación de la información con fines de investigación. Siempre será mejor tener una pregunta valiosa, incluso cuando se responda con un diseño con mayor riesgo de sesgos, que una pregunta irrelevante o sin aplicabilidad.

PALABRAS CLAVE: Diseños. Pesquisa. Ensamble. Maniobra. Sesgos.

\begin{abstract}
Research designs refer to the way information is obtained and are limited by ethical, economic and temporal viability. Research designs are standardized strategies to reduce biases, which in the architectural model of research are identified in the baseline state, the maneuver and the outcome; hence, there are no specific designs for each question. The design with the lowest probability of bias is the clinical trial, followed by cohort and case-control studies and, finally, by cross-sectional surveys. Among the main characteristics that give merit to research designs are the following: population inquiry, which refers to the situation of the population in relation to the clinical course/natural history of the disease; the maneuver, or action that is expected to modify the baseline state, which can be observational or experimental; follow-up, or documented monitoring that is given to each subject, which can be longitudinal or cross-sectional; and directionality, which can prolective or retrolective and refers to the timing of data collection for research purposes. It will always be better having a valuable question, even when answered with a design with higher risk of bias, than a question that is irrelevant or has no applicability.
\end{abstract}

KEY WORDS: Designs. Inquiry. Assembly. Maneuver. Biases.

Correspondencia:

*Juan O. Talavera

E-mail: jotalaverap@abchospital.com
Fecha de recepción: 15-04-2019

Fecha de aceptación: 17-05-2019

DOI: $10.24875 / G M M .19005226$
Gac Med Mex. 2019;155:399-405

Disponible en PubMed www.gacetamedicademexico.com 


\section{Introducción}

No existe un diseño específico para cada pregunta. Los diseños se refieren a la forma de recolección de la información y se determinan por la factibilidad ética, económica y temporal.

Una vez revisado el objetivo y justificación de cualquier proyecto 0 artículo de investigación, el primer apartado que se aborda es el de métodos, que se refiere al diseño de investigación, característica que permite identificar cómo se recolectó o recolectará la información. ${ }^{1,2}$ Jerárquicamente, de mayor a menor calidad de información, se encuentran el ensayo clínico, seguido de la cohorte, el estudio de casos y controles $y$, finalmente, la encuesta transversal. ${ }^{3} \mathrm{~A}$ partir de estos existen varios diseños que combinan características, como los casos y controles anidados en una cohorte ${ }^{4} \mathrm{o}$ los estudios longitudinales, creados a partir de encuestas transversales, ${ }^{5}$ entre muchas posibles combinaciones, incluso, puede hablarse de una encuesta transversal anidada en una cohorte.

Las diferentes formas de recolección de la información buscan evitar los sesgos en los distintos componentes del modelo arquitectónico de la investigación ${ }^{6}$, ya sea en el estado basal, en la maniobra o en el resultado (Figura 1):

- En el estado basal debe considerarse la demarcación diagnóstica, con el fin de evitar un "ensamble inadecuado", y la demarcación pronóstica, para atenuar el "sesgo de susceptibilidad pronóstica".

- Durante la maniobra hay que considerar la calidad de la maniobra principal, la presencia de maniobras periféricas y el manejo de eventos adversos, para disminuir el "sesgo de ejecución".

- Hay que garantizar la idéntica medición del desenlace en todos los individuos sin distinción alguna, mucho menos dependiente del tipo de maniobra, así como la igualdad en tiempos y procedimiento en cada medición, con el fin de evitar el "sesgo de detección".

- Es necesario documentar las pérdidas durante el seguimiento, de tal forma que se pueda evaluar la similitud de características entre los sujetos perdidos y los que continúan hasta el final del estudio, y así ponderar la presencia del "sesgo de transferencia".

\section{Características de los diseños de investigación (Tabla 1)}

Cabe resaltar que la pesquisa de la población es la característica que más determina errores en los diseños de investigación. La pesquisa de la población se refiere al momento dentro del fenómeno de causalidad en el que se considera a la población candidata a participar. Es así que puede realizarse concordante con el estado basal, como ocurre en los diseños de cohorte experimental (ensayo clínico) u observacional, o discordante con el estado basal, como ocurre en el diseño de casos y controles o en la encuesta transversal, en los que se busca la población candidata una vez que el desenlace ha ocurrido. Mientras la pesquisa de la población va dirigida al universo de donde provienen todos los candidatos por evaluar, el ensamble de la población lo conforman los sujetos que, habiendo cubierto el criterio de salud o enfermedad de interés (criterios diagnósticos), reúnen los criterios de selección; ${ }^{6}$

De los otros componentes propios del diseño de investigación resaltaremos tres:

- Exposición a la maniobra. Acción que se espera modifique la condición basal del paciente provocando un cambio que se conoce como resultado. Cuando la maniobra es asignada como parte de la investigación de interés se le conoce como experimental, lo que caracteriza al ensayo clíni$\mathrm{co}^{7,8}$ Cuando la maniobra tuvo razones para su aplicación ajenas a la investigación, y en cuyo caso posteriormente se identifica dicha aplicación previa, pero ahora con fines de investigación, se le considera una maniobra observacional (por ejemplo: tabaquismo como causa autoseleccionada y edad como causa por acción de la naturaleza, e incluso el uso de un fármaco por recomendación o automedicación). Debido a esta característica, los diseños de cohorte, casos y controles y encuesta transversal se les conoce como estudios observacionales..$^{9,10}$

- Seguimiento. Se refiere al monitoreo que se da a cada sujeto en relación con el curso clínico o historia natural de la enfermedad. Se reconoce un estudio como longitudinal cuando existe evidencia documentada en tiempo real de cada uno de los componentes dentro del fenómeno de causalidad, se hayan registrado o no con fines de investigación; inicialmente se registra o se debió haber registrado el estado basal, luego, conforme transcurre el tiempo, la maniobra y, finalmente, el desenlace. Entre las razones ajenas a la investigación que pudiesen haber dado origen al registro están la atención médica, la revisión médica periódica, etcétera. ${ }^{11}$ 


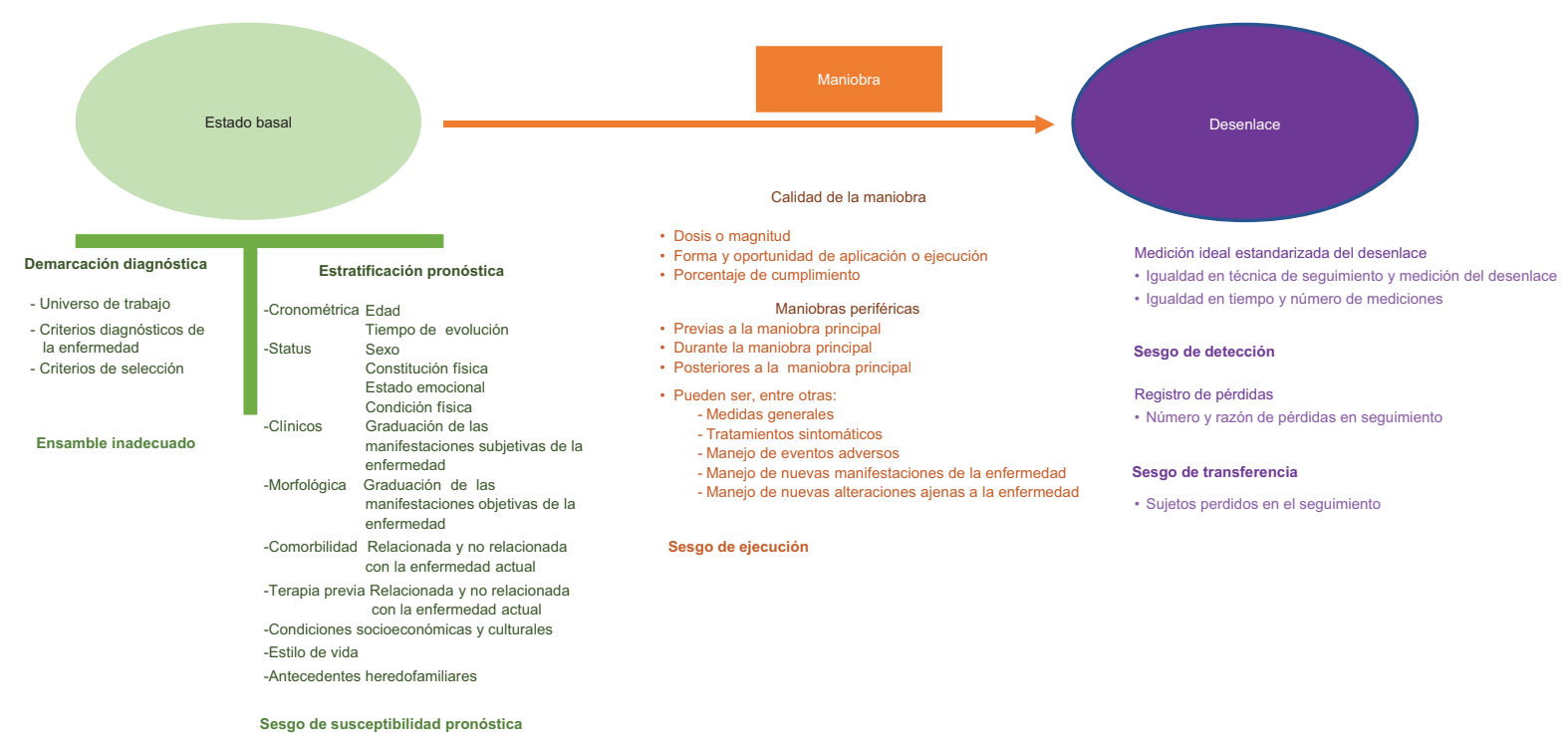

Figura 1. Modelo arquitectónico de la investigación.

Tabla 1. Características principales de los diseños de investigación

\begin{tabular}{|c|c|c|c|c|c|}
\hline $\begin{array}{l}\text { Diseño de } \\
\text { Investigación }\end{array}$ & $\begin{array}{l}\text { Pesquisa de la } \\
\text { población }\end{array}$ & Maniobra & $\begin{array}{l}\text { Seguimiento } \\
\text { del individuo }\end{array}$ & $\begin{array}{l}\text { Direccionalidad de la } \\
\text { información }\end{array}$ & Descripción de característica \\
\hline $\begin{array}{l}\text { Ensayo clínico } \\
\text { (cohorte } \\
\text { experimental) }\end{array}$ & $\begin{array}{l}\text { Concurrente al } \\
\text { estado basal } \\
\text { (tiempo 0) }\end{array}$ & Experimental & Longitudinal & Prolectivo & \multirow{4}{*}{$\begin{array}{l}\text { Diseño de investigación, forma como se } \\
\text { obtiene la información, "calidad de la } \\
\text { información". } \\
\text { Pesquisa de la población, momento dentro del } \\
\text { curso clínico/historia natural de la enfermedad } \\
\text { en que se busca la población. } \\
\text { Maniobra, acción que se espera modifique la } \\
\text { condición basal: 1. Experimental, maniobra } \\
\text { asignada con fines de investigación. } \\
\text { 2. Observacional, maniobra presente por } \\
\text { causas ajenas a la investigación (por } \\
\text { ejemplo: por acción de la naturaleza [edad], } \\
\text { autoseleccionada [tabaquismo], por otras } \\
\text { indicaciones [atención médica]). } \\
\text { Seguimiento, se refiere al monitoreo que se } \\
\text { da a cada sujeto en relación con el curso } \\
\text { clínico o historia natural de la enfermedad. } \\
\text { 1. Longitudinal, existe evidencia documentada } \\
\text { del estado basal y maniobra antes del } \\
\text { desenlace. 2. Transversal, el paciente es } \\
\text { evaluado en forma estacionaria; tanto estado } \\
\text { basal, maniobra y desenlace se miden a un } \\
\text { solo tiempo. } \\
\text { Direccionalidad de la información, momento } \\
\text { de recolección de la información con fines } \\
\text { de investigación; } 1 \text {. Prolectivo, en tiempo } \\
\text { real, cuando el fenómeno está ocurriendo. } \\
\text { 2. Retrolectivo, se recopila la información } \\
\text { cuando el fenómeno ya ocurrió. Pudo haber } \\
\text { sido registrada la información en tiempo real } \\
\text { con anterioridad, pero por razones ajenas a la } \\
\text { investigación. }\end{array}$} \\
\hline $\begin{array}{l}\text { Cohorte } \\
\text { (cohorte } \\
\text { observacional) }\end{array}$ & $\begin{array}{l}\text { Concurrente al } \\
\text { estado basal } \\
\text { (tiempo 0) }\end{array}$ & Observacional & $\begin{array}{l}\text { Longitudinal } \\
\text { longitudinal }\end{array}$ & $\begin{array}{l}\text { Prolectivo } \\
\text { Retrolectivo (histórica) }\end{array}$ & \\
\hline $\begin{array}{l}\text { Casos y } \\
\text { controles }\end{array}$ & $\begin{array}{l}\text { Divergente al } \\
\text { estado basal } \\
\text { (tiempo 3) }\end{array}$ & Observacional & $\begin{array}{l}\text { Transversal/ } \\
\text { longitudinal }\end{array}$ & Retrolectivo & \\
\hline $\begin{array}{l}\text { Encuesta } \\
\text { transversal }\end{array}$ & $\begin{array}{l}\text { Divergente } \\
\text { al estado } \\
\text { basal (tiempo 3) }\end{array}$ & Observacional & $\begin{array}{l}\text { Transversal/ } \\
\text { longitudinal }\end{array}$ & Retrolectivo & \\
\hline
\end{tabular}


documentada en el momento real en que ocurrieron los hechos, en forma artificial debe intentarse reconstruir la temporalidad real de cada apartado, por ejemplo: en un paciente diabético con cinco años de evolución en quien se quiere estudiar daño a órgano blanco como consecuencia del control metabólico, se puede reconstruir su estado basal preguntándole su situación cinco años atrás, tanto los criterios que en su momento se hubieran aplicado, como sus características basales y el control que ha experimentado de entonces hasta el momento en que se realiza el estudio. De esta forma, los pacientes que en la reconstrucción de sus características no eran candidatos para entrar al estudio no deberán ser incluidos; de igual forma, será necesario tratar de identificar en qué momento pudieron haber sufrido daño a órgano blanco de documentarse en el momento actual. Afortunadamente, el desarrollo tecnológico ha facilitado la existencia de registros que por distintas razones monitorean el estado de salud, lo que incrementa el seguimiento en tiempo real de muchas personas que posteriormente serán sujetos de estudio de investigación; con ello, el seguimiento longitudinal se ha vuelto y continuará haciéndose cada vez más común. Este hecho incrementará la calidad de la información, reduciendo los errores de medición inherentes a la memoria. ${ }^{12}$ El único caso en el que no existen estos errores es en la descripción del estado basal de pacientes con una enfermedad de reciente inicio (o cualquier estado de reciente inicio), donde no se busca asociación alguna, sino solo caracterizar dichas condiciones basales, sin embargo, una vez recabada la información lo común es que se realicen comparaciones entre el estado basal y los tiempos subsecuentes. ${ }^{13}$ Direccionalidad del estudio. Momento de recolección de la información con fines de investigación. Un estudio se considera prolectivo cuando la recolección se realiza en tiempo real y con fines de investigación al momento en que el fenómeno de causalidad está ocurriendo; se documenta inicialmente con fines de investigación el estado basal, posteriormente la maniobra y, en su momento, el desenlace. ${ }^{14}$ Un estudio es retrolectivo cuando se recopila la información con fines de investigación una vez que el fenómeno de causalidad ya ocurrió; incluye los casos en los que la información se recolectó en tiempo real —cuando el fenómeno de causalidad está ocurriendo-, pero con fines ajenos a investigación, como en la atención médica o la revisión médica. ${ }^{15}$

\section{Diseños de investigación ${ }^{16,17}$}

En el ensayo clínico, también conocido como cohorte experimental, al igual que en la cohorte prospectiva, la recolección de la información correspondiente a los distintos componentes del fenómeno de causalidad, se realiza en tiempo real y con fines de investigación. Por su parte, en la cohorte histórica o retrospectiva, la recolección de la información en tiempo real se llevó a cabo con fines ajenos a la investigación; generalmente la recolección es realizada en su momento con el objetivo de dar atención médi$\mathrm{ca}^{18} \mathrm{o}$ durante la revisión médica, ${ }^{19}$ entre otros.

Como se mencionó, en estos diseños existe una concordancia entre la pesquisa de la población y el ensamble de los candidatos. En el ensayo clínico existe asignación de la maniobra con fines de investigación y en la cohorte solo se mide la exposición a la maniobra. En ambos, el seguimiento es longitudinal $^{20}$ (Figura 2).

Frecuentemente se cometen errores y diseños que no corresponden se asumen como cohortes retrospectivas o históricas, por ejemplo: algunos estudios se refieren como cohorte cuando en realidad se trata de series de casos porque la pesquisa de la población se realizó partiendo del desenlace, en consecuencia resulta discordante con el ensamble de la población (el ensamble siempre se realiza en el estado basal) y solo se cuenta con una porción de la cohorte original, que conforma una población de sobrevivientes que corresponde a una serie de casos de sobrevivientes. ${ }^{21,22} \mathrm{Si}$ la población candidata se busca en el registro de pacientes asistentes a atención médica en el momento de la investigación, se habrán dejado fuera todos los pacientes que murieron o que dejaron de asistir a atención médica por cualquier razón en el periodo previo entre lo que fue su estado basal y el momento en el que se lleva a cabo la investigación. ${ }^{23}$ La sobreestimación del pronóstico y de los beneficios de las terapias son problemas frecuentes derivados de este tipo de diseños.

Una forma de evitar el error mencionado y formar una cohorte histórica es mantener en mente la pesquisa de la población y su concordancia con el ensamble. Para ello es importante investigar la población en una fecha previa cuyos años cuantificados hasta el momento actual sean el promedio de seguimiento que se desea para dicha cohorte; es decir, si se desea 


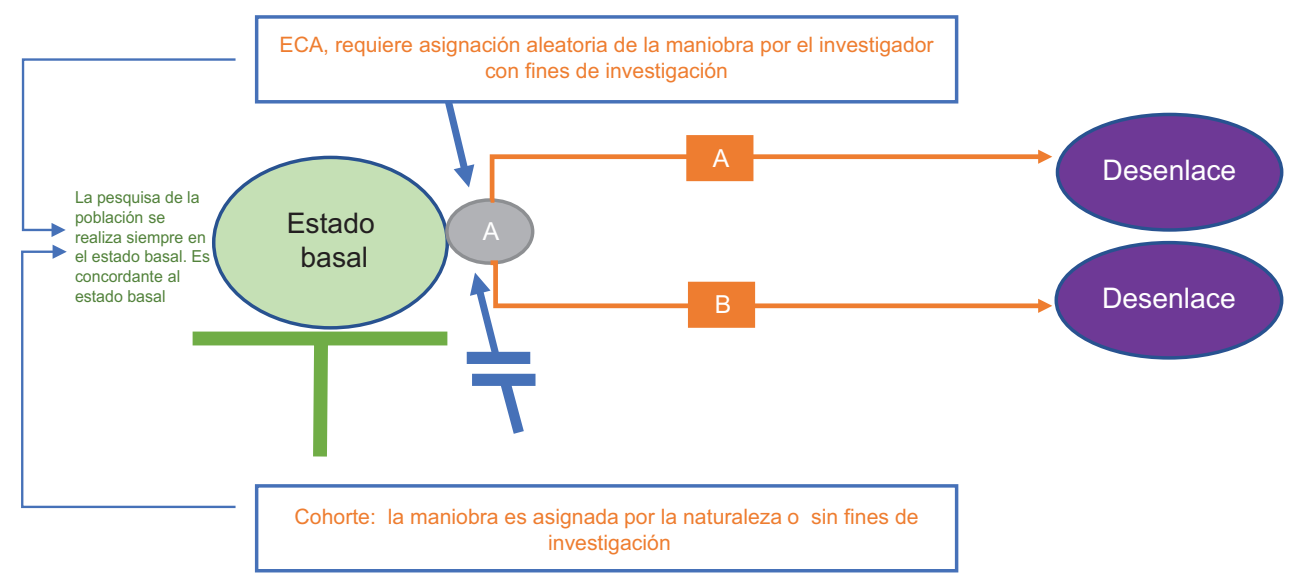

Figura 2. Diseño de estudios longitudinales (cohortes o ensayo clínico aleatorizado) y sus principales sesgos. ECA = ensayo clínico aleatorizado, $A$ = asignación aleatoria por el investigador.

estudiar la incidencia de daño a órgano blanco en diabéticos a 10 años de evolución, en lugar de buscarlos en registros de la consulta actual (2019), habrá que buscarlos en el registro de ingreso de pacientes de reciente diagnóstico de 10 años atrás (2009). Una vez identificados, se reconstruye el fenómeno de causalidad, empezando por el ensamble, la estratificación pronóstica, la maniobra y maniobras periféricas, y así sucesivamente hasta llegar al desenlace. De esta manera podrá identificarse a los pacientes que murieron o se perdieron durante el seguimiento y se sabrá en qué condición se encontraban en su última evaluación, sucesos que se desconocen cuando erróneamente se considera como cohorte lo que es una serie de casos donde solo están los sobrevivientes. ${ }^{24}$

\section{Casos y controles ${ }^{25}$}

El estudio de casos y controles, como su nombre lo indica, lleva a cabo la pesquisa de población candidata a partir del desenlace, donde selecciona a un grupo de casos (sujetos ya con el desenlace) y a un grupo de controles (sujetos sin el desenlace), en consecuencia se trata de una recopilación retrospectiva de la información con fines de investigación, ${ }^{26,27}$ aun cuando existe la posibilidad de que dicha información haya sido recolectada en tiempo real, pero con fines ajenos a investigación (expediente clínico, cartilla de vacunación y revisión médica, entre otras fuentes).

Posteriormente, en forma artificial se reconstruye la temporalidad de ocurrencia del fenómeno de causalidad. Es decir, una vez que se identifica la población candidata, aunque artificial, la reconstrucción del fenómeno de causalidad se realiza ya sea a través de algún registro existente o la memoria del paciente 0 familiares, evaluando los criterios de selección al momento en que existió temporalmente el estado basal (que como en el ejemplo anterior pudo haber sido 10 años atrás), y de igual forma se reconstruye la exposición de la maniobra al momento subsecuente correspondiente y así hasta la evaluación del desenlace. De esta forma se intenta reconstruir la temporalidad del fenómeno real, aunque en forma artificial; frecuentemente se confunde la pesquisa con el ensamble de la población y se aplican los criterios de selección erróneamente al momento de la pesquisa y en consecuencia se quebrantan los principios de causalidad. No debemos olvidar que si bien la pesquisa de la población en el estudio de casos y controles es en el desenlace, el ensamble se realiza a lo que fue en el estado basal (Figura 3).

El hecho de que la pesquisa se realice en el desenlace provoca el empleo de una población de sobrevivientes y frecuentemente de un origen no específico - los sobrevivientes pueden provenir de distintas poblaciones de origen- Si bien puede haber sesgos de distinta magnitud por la calidad de la información en la reconstrucción de lo sucedido tiempo atrás (siempre será mejor si proviene de un expediente que de la memoria), nunca podrá evitarse la existencia del sesgo de transferencia, que dependerá de la movilidad y mortalidad de la población de donde proceden los sujetos estudiados que corresponden al grupo de sobrevivientes. ${ }^{28-30}$

\section{Encuesta transversa/ ${ }^{31}$}

En la encuesta transversal, la pesquisa de la población (al igual que en el estudio de casos y controles) se realiza cuando existe la posibilidad de encontrar 


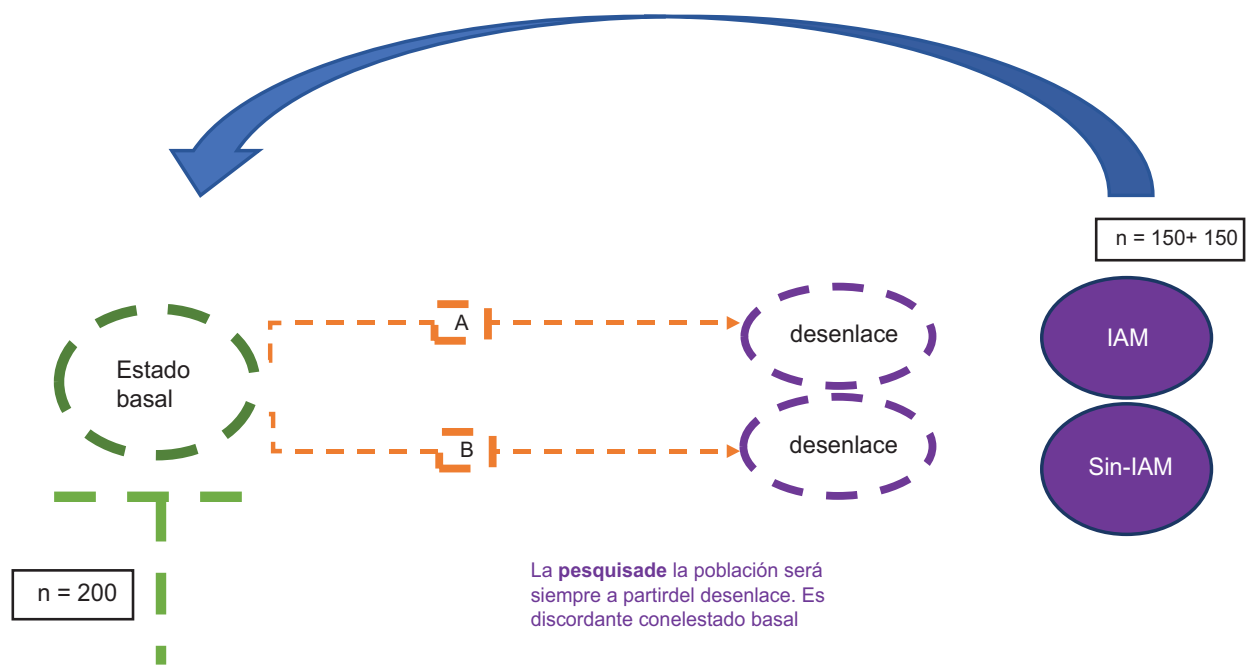

Figura 3. Casos y controles. La pesquisa solo ofrece a la población candidata, después esta población tiene que evaluarse siguiendo el pensamiento lógico de causalidad, es decir, se parte del estado basal en dirección al desenlace. Se aplican los criterios de selección al estado basal, tal y como se construye el diseño arquitectónico, y aquellos casos y controles no incluidos (no seleccionados) deberán ser sustituidos en número hasta alcanzar el tamaño de muestra.

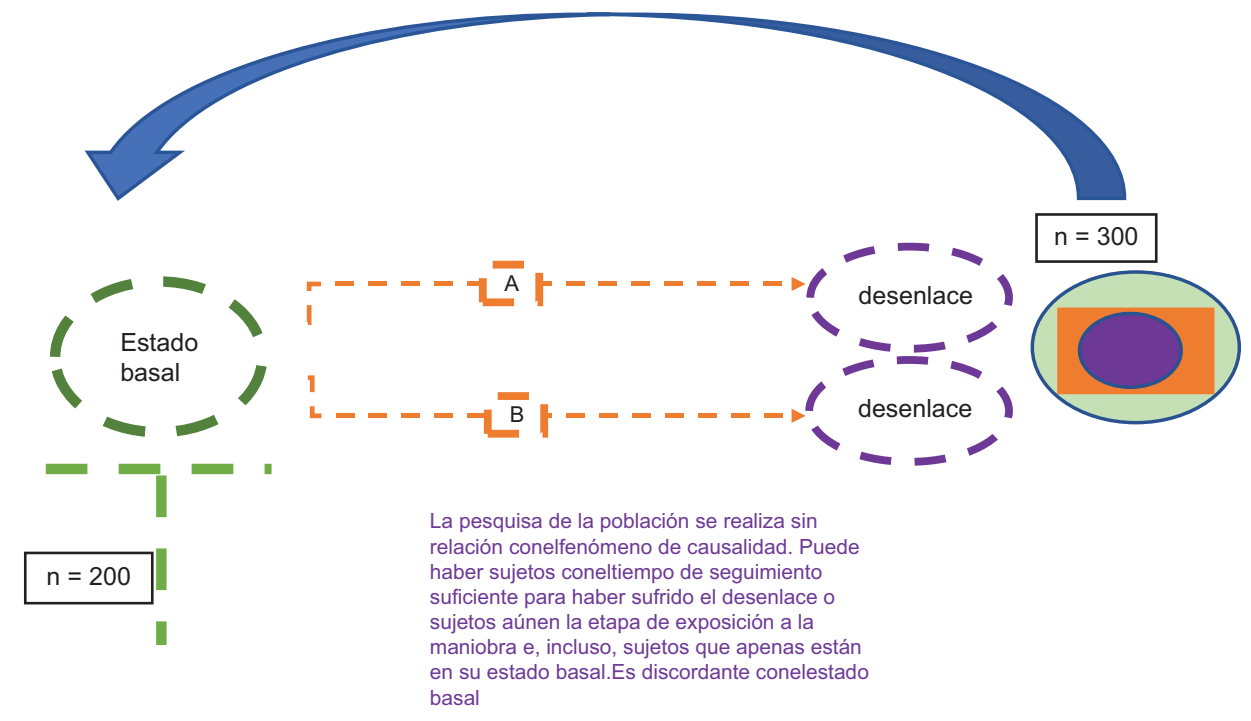

Figura 4. Encuesta transversal. La pesquisa solo ofrece a la población candidata, después esta población tiene que evaluarse siguiendo el pensamiento lógico de causalidad, es decir, se parte del estado basal en dirección al desenlace. Se aplican los criterios de selección al estado basal, tal y como se construye en el modelo arquitectónico, y los sujetos no incluidos (no seleccionados) deberán ser sustituidos en número hasta alcanzar el tamaño de muestra.

sujetos con el desenlace de interés, aun cuando no se busca a una población específicamente por la presencia o ausencia del desenlace. En este caso se tendrán los sujetos con el desenlace de acuerdo con la prevalencia del mismo en dicha población. En la encuesta transversal se pueden encontrar sujetos en cualquier etapa del fenómeno de causalidad, es decir, habrá sujetos cuyo curso clínico o historia natural de la enfermedad se ubica en el tiempo en el que puede existir el desenlace $y$, en consecuencia, se encontrarán sujetos con y sin el desenlace, otros se encontrarán en el periodo de exposición a la maniobra y otros tal vez en el inicio de la condición basal de interés (Figura 4).

Al reconstruir en forma artificial el fenómeno de causalidad se retrocede en el tiempo para obtener la información del estado basal, la maniobra y el desenlace, similar a lo referido en el estudio de casos y controles. Al igual que en este, los sujetos incluidos pueden provenir de múltiples poblaciones y nuevamente se trata solo de sobrevivientes, en 
consecuencia, se corre el riesgo de sesgo de transferencia. En ocasiones se refieren estudios de encuesta transversal sin el objetivo de realizar comparaciones pensando que se evitan sesgos, sin embargo, habría que establecer en qué momento del curso clínico o historia natural de la enfermedad se encuentran los individuos y caracterizar los que están en su estado basal de los que están en la etapa de exposición de la maniobra o desenlace. No hay forma de evitar el sesgo de transferencia. ${ }^{32}$

\section{Conclusiones}

Los diseños de investigación describen la forma de recolección de la información, no existe un diseño específico para cada pregunta de investigación. Esta conserva la estructura del pensamiento causal, el cual a su vez se aborda mediante el modelo arquitectónico de la investigación.

Una misma pregunta puede ser contestada por cualquiera de los diseños. Los diseños prospectivos con pesquisa en el estado basal -ensayo clínico y cohorte prospectiva- son los de mayor calidad de información, sin embargo, frecuentemente las limitaciones económicas, éticas y de tiempo obligan a considerar diseños retrospectivos. Dentro de estos últimos, la cohorte histórica (cuya pesquisa se realiza en el estado basal) resulta la mejor, disminuyendo los sesgos de transferencia propios de los diseños retrospectivos con pesquisa discordante al estado basal (estudio de casos y controles y encuesta transversal).

Siempre será mejor contar con una pregunta valiosa, aun cuando esta sea contestada con un diseño de menor calidad, que contar con una pregunta irrelevante contestada con un diseño de mayor calidad. Aquello que no tiene relevancia o aplicabilidad, no tiene sentido en la investigación clínica.

\section{Bibliografía}

1. Talavera JO. Research designs. Rev Med Inst Mex Seguro Soc 2013:51:S10-S15.

2. Hernández-Sampieri R. Metodología de la investigación. México: McGraw Hill; 2014

3. Murad MH, Asi N, Alsawas M, Alahdab F. New evidence pyramid. Evid Based Med. 2016:21:125-127.

4. Ernster VL. Nested case-control studies. Prev Med. 1994;23:587-590.

5. Rivas-Ruiz R, Clark P, Talavera JO, Huitrón G, Tamayo JA, Salmerón J. Bone speed of sound throughout lifetime assessed with quantitative ultrasound in a Mexican population. J Clin Densitom. 2015;18:68-75.

6. Talavera JO, Wacher-Rodarte NH, Rivas-Ruiz R. Investigación clínica III. Estudios de causalidad. Rev Med Inst Mex Seguro Soc. 2011;49:289-294.

7. ASCEND Study Collaborative Group, Bowman L, Mafham M, Wallendszus K, Stevens W, Buck G, et al. Effects of n-3 fatty acid supplements in diabetes mellitus. N Engl J Med. 2018;379:1540-1550.

8. Salminen $\mathrm{P}$, Paajanen $\mathrm{H}$, Rautio T, Nordström P, Aarnio M, Rantanen T, et al. Antibiotic therapy vs appendectomy for treatment of uncomplicated acute appendicitis: the APPAC Randomized Clinical Trial. JAMA. 2015:313:2340-2348.

9. Méndez-Hernández P, Flores Y, Siani C, Lamure M, Dosamantes-Carrasco LD, Halley-Castillo $E$, et al. Physical activity and risk of metabolic syndrome in an urban Mexican cohort. BMC Public Health. 2009:9:276.

10. Diez-Ruiz A, Bueno-Errandonea A, Núñez-Barrio J, Sánchez-Martín I, Vrotsou K, Vergara I. Factors associated with frailty in primary care: a prospective cohort study. BMC Geriatr. 2016;28:91

11. Ventura-Ríos L, Bañuelos-Ramírez D, Hernández-Quiroz-MC, Robles-San Román M, Irazoque-Palazuelos F, Goycochea-Robles MV. Patient survival and safety with biologic therapy. Results of the Mexican National Registry Biobadamex 1.0. Reumatol Clin. 2012;8:189-194.

12. Machado AD, Anjos FSND, Domingos MAM, Molina MDCB, Marchioni DML, Benseñor IJM, et al. Dietary intake of non-dialysis chronic kidney disease patients: the PROGREDIR study. A cross-sectional study. Sao Paulo Med J. 2018;136:208-215.

13. Perminow G, Brackmann S, Lyckander LG, Franke A, Borthne A, Rydning $A$, et al. A characterization in childhood inflammatory bowel disease, a new population-based inception cohort from South-Eastern Norway, 2005-07, showing increased incidence in Crohn's disease. Scand J Gastroenterol. 2009:44:446-456.

14. Gallegos-Carrillo K, Flores YN, Denova-GutiérrezE, Méndez-HernándezP, Dosamantes-Carrasco LD, Henao-Morán S, et al. Physical activity and reduced risk of depression: results of a longitudinal study of Mexican adults. Health Psychol. 2013:32:609-615.

15. Pacheco NL, Andersen AM, Kamper-Jørgensen M. Preeclampsia and breast cancer: the influence of birth characteristics. Breast. 2015;24: 613-617.

16. Talavera JO, Rivas-Ruiz R. Investigación clínica IX. Del juicio clínico al ensayo clínico. Rev Med Inst Mex Seguro Soc. 2013;51:S58-S63.

17. Talavera JO, Rivas-Ruiz R. Investigación clínica X. Del juicio clínico al diseño de cohorte. Rev Med Inst Mex Seguro Soc. 2013;51:S64-S69.

18. Reynoso-Noverón N, Villarreal-Garza C, Soto-Pérez de Celis E, Arce-Salinas C, Matus-Santos J, Ramírez-Ugalde MT, et al. Clinical and epidemiological profile of breast cancer in Mexico: results of the Seguro Popular. J Glob Oncol. 2017;3:757-764

19. Briseño-Bass $P$, Chávez-Pérez R, López-Zendejas $M$. Prevalence of liver steatosis and its relation to liver function tests and lipid profile in patients at medical check-up. Rev Gastroenterol Mex. 2018;3:S0375-S0906.

20. Cantoral A, Téllez-Rojo MM, Ettinger AS, Hu H, Hernández-Ávila M, Peterson K. Early introduction and cumulative consumption of sugar-sweetened beverages during the pre-school period and risk of obesity at 8-14 years of age. Pediatr Obes. 2016;11:68-74

21. Cacho-Díaz B, Spínola-Maroño H, Reynoso N, González-Aguilar A, Mohar-Betancourt A. Role of overweight, obesity, and comorbidities in the prognosis of patients with breast cancer with brain metastases. Clin Breast Cancer. 2019;19:394-398.

22. Espiritu Al, Mesina BVQ, Puerto AAD, Reyes NGD, Damian LF, Pascual V JLR. Neuromyelitis optica spectrum disorder in a tertiary hospital in the Philippines: a case series. Mult Scler Relat Disord. 2019;31: 124-130.

23. Abdelmabood S, Fouda AE, Boujettif F, Mansour A. Treatment outcomes of children with acute lymphoblastic leukemia in a middle-income developing country: high mortalities, early relapses, and poor survival. $\mathrm{J} \mathrm{Pe}-$ diatr (Rio J). 2018;S0021-7557:30583-30587.

24. Lepe-Zúñiga JL, Méndez-Cigarroa AO, Jerónimo-López FJ, Hernández-Orantes JG. [Sobrevida global de pacientes con leucemia aguda en el Hospital de Especialidades Pediátricas de Chiapas, México]. Bol Med Hosp Infant Mex. 2018;75(6):338-351

25. Talavera JO, Rivas-Ruiz R. From clinical judgment to case-control design. Rev Med Inst Mex Seguro Soc. 2013;51:S70-S75.

26. Doll R, Hill AB. A study of the aetiology of carcinoma of the lung. Br Med J. 1952;2:1271-1286.

27. Girma S, Fikadu T, Abdisa E. Maternal common mental disorder as predictors of stunting among children aged 6-59 months in western Ethiopia: a case-control study. Int J Pediatr. 2019;2019:4716482.

28. Di Forti M, Quattrone D, Freeman TP, Tripoli G, Gayer-Anderson C, Quigley $\mathrm{H}$, et al. The contribution of cannabis use to variation in the incidence of psychotic disorder across Europe (EU-GEI): a multicentre case-control study. Lancet Psychiatry. 2019:6:427-436.

29. Hodge SE, Subaran RL, Weissman MM, Fyer AJ. Designing case-control studies: decisions about the controls. Am J Psychiatry. 2012;169:785-789.

30. Blaser L, Hassna H, Hofmann S, Holbro A, Haschke M, Zeller A, et al. Leucopenia associated with metamizole: a case-control study. Swiss Med Wkly. 2017;147:w14438

31. Talavera JO, Rivas-Ruiz R. From clinical judgment to cross-sectional survey. Rev Med Inst Mex Seguro Soc. 2013;51:S76-S79.

32. Shigdel R, Stubbs B, Sui X, Ernstsen L. Cross-sectional and longitudinal association of non-exercise estimated cardiorespiratory fitness with depression and anxiety in the general population: the HUNT study. J Affect Disord. 2019;252:122-129. 